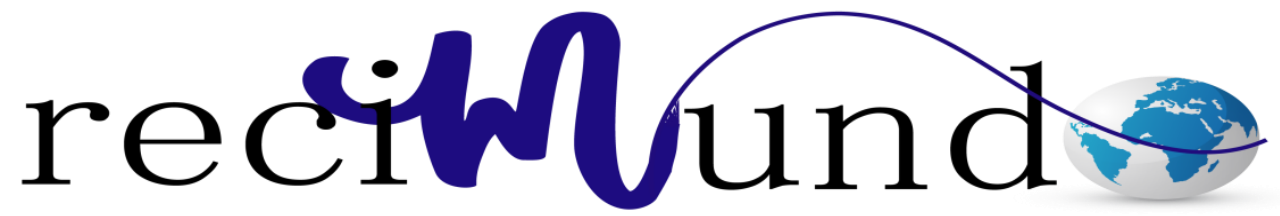

Revista Científica Mundo de la Investigación y el Conocimiento

Ruth Cecibelt Cedeño Álvarez ${ }^{\text {a }}$; José Bladimir Guarnizo Delgado ${ }^{\text {}}$; Iván Santiago Pacheco Proaño ${ }^{c}$

Adopción del recurso teatral en el tratamiento didáctico del proceso de enseñanza aprendizaje devenido experiencia axiológica

Revista Científica Mundo de la Investigación y el Conocimiento. Vol. 2 núm.2, mayo, ISSN: 2588-073X, 2018, pp. 761-781

DOI: 10.26820/recimundo/2.(2).2018.761-781

Editorial Saberes del Conocimiento

Recibido: 05/12/2017

Aceptado: $15 / 02 / 2018$

a. Docente, Universidad Estatal de Bolívar, Guaranda-Bolívar-Ecuador; cecycedeno1996@yahoo.es

b. Docente, Universidad Estatal de Bolívar, Guaranda-Bolívar-Ecuador; jblady_guarnizo@yahoo.es

c. Docente, Universidad Técnica de Cotopaxi; ivan.pacheco@utc.edu.ec 


\section{Adopción del recurso teatral en el tratamiento didáctico del proceso de enseñanza aprendizaje devenido experiencia axiológica}

Vol. 2, núm. 2., (2018)

Ruth Cecibelt Cedeño Álvarez; José Bladimir Guarnizo Delgado; Iván Santiago Pacheco Proaño

\section{RESUMEN}

El presente artículo resume los resultados de la investigación devenida experiencia axiológica, y desarrollada a partir de la inclusión en la malla curricular de la carrera de Comunicación Social, Universidad Estatal de Bolívar, Ecuador- taller de estudio y creación teatral. El objetivo alcanzado fue el de fomentar una cultura contra la violencia, proyectada en pieza teatral abordando desde la perspectiva educativa-cultural el asunto. Esta investigación enriquece el tratamiento didáctico del proceso de enseñanza - aprendizaje de la citada asignatura para lo cual se adopta modelo formativo y metodología de carácter cualitativo.

Palabras Claves: Recurso teatral; experiencia axiológica; proceso enseñanza aprendizaje; modelo formativo. 


\title{
Adopción del recurso teatral en el tratamiento didáctico del proceso de enseñanza aprendizaje devenido experiencia axiológica
}

Vol. 2, núm. 2., (2018)

Ruth Cecibelt Cedeño Álvarez; José Bladimir Guarnizo Delgado; Iván Santiago Pacheco Proaño

\begin{abstract}
The present article summarizes the research findings that happened axiological experience, and developed from the inclusion in the curricular net of the career of Social Communication, becoming study workshop of theatrical creation - State Bolivar's University, Ecuador -. The attained objective was the one of promoting a culture against violence, projected in play discussing from the educational cultural perspective the subject. This investigation enriches the didactic treatment of the process of teaching - learning of the quoted subject of study for which formative model and methodology of qualitative character are embraced.
\end{abstract}

Key words: Theatrical resource; axiological experience; teaching learning process; formative model. 


\section{Adopción del recurso teatral en el tratamiento didáctico del proceso de enseñanza aprendizaje devenido experiencia axiológica}

Vol. 2, núm. 2., (2018)

Ruth Cecibelt Cedeño Álvarez; José Bladimir Guarnizo Delgado; Iván Santiago Pacheco Proaño

\section{Introducción.}

El recurso teatral en el contexto educacional, constituye parte de las técnicas pedagógicas utilizadas para desarrollar las capacidades de expresión y comunicación de los estudiantes, desarrollando no solamente en habilidades del lenguaje como leer, escribir, escuchar o hablar, sino esencialmente, en la habilidad de comunicar.

Como recurso didáctico, ha sido empleado en varias épocas, desde los diálogos teatrales recreados por los humanistas para la enseñanza del latín en las universidades renacentistas, hasta las modernas técnicas de dramatización que descubren la enseñanza como un todo, en las cuales teoría y práctica se retroalimentan y se contemplan tanto los aspectos cognitivos de la formación como acertadamente plantea Meléndez (2013, p. 12), "los aspectos afectivos, perceptivos, psicomotores, artísticos y sociales" (p. 12), significando el aspecto axiológico como un parámetro clave en esta investigación.

Este artículo describe la experiencia pedagógica - teatral de los docentes y estudiantes de la Universidad Estatal de Bolívar, ubicada en la provincia homónima de Ecuador, quienes desarrollaron un taller teatral que culminó con la presentación de la obra titulada "El coleccionista de senos" en varias sedes de la ciudad de Chimbo; esta puesta en escena fue promovida por la profesora que le imparte la asignatura de Lenguaje y Comunicación al grupo de IV ciclo de la carrera de Comunicación Social y autora principal de ese artículo. 


\section{Adopción del recurso teatral en el tratamiento didáctico del proceso de enseñanza aprendizaje devenido experiencia axiológica}

Vol. 2, núm. 2., (2018)

Ruth Cecibelt Cedeño Álvarez; José Bladimir Guarnizo Delgado; Iván Santiago Pacheco Proaño

El texto inédito es creación del autor José Vizcaíno; es un retrato de la sociedad moderna bolivarense en donde la fábula como recurso trasmite el mensaje de no a la violencia, en todas sus manifestaciones. Está estructurada en cinco escenas principales. La presente investigación alcanzó el objetivo de fomentar una cultura contra la violencia, fenómeno que afecta a la sociedad ecuatoriana y al resto de los países del área centro y sur americana y se utilizó como vehículo para alcanzar este propósito el desarrollo de un taller de creación teatral.

Esta experiencia axiológica contó con un referente en la ciudad española de Sevilla, donde se proyectó taller de teatro para lograr el respeto a la diversidad. Su autor Gaspar Rodríguez Aparicio, describió su metodología apoyado desde la psicología de Ausubel (1975) y Vygotsky (1978), quienes evocan a la participación colectiva y activa; el corresponder a su autoformación desde la actividad; el papel lúdico y creativo del teatro en el aprendizaje evolutivo del individuo. (Rodríguez, 2009, p. 89)

Otros autores, referentes teóricos de este trabajo, son ubicados a continuación según las aportaciones teóricas y las esferas del conocimiento a que pertenecen. Los partidarios de introducir la enseñanza del teatro en el currículum escolar forman el grupo sucesivo: O'Neill (1995), Froese (1996), Edwards (1997), Wagner (1998), Baldwin, Fleming y Neelands (2003), Laferrière y Motos (2003) y Navarro (2006).

Los autores que orientaron desde sus investigaciones esta experiencia para establecer los principios básicos del teatro: Brook (2001); para aplicar el modelo de decisión individual de participación en eventos artísticos: McCarthy y Jinnett (2001); para desarrollar un modelo formativo: González (2012); para adecuar los retos del teatro en la escuela: Motos (2009). 


\section{Adopción del recurso teatral en el tratamiento didáctico del proceso de enseñanza aprendizaje devenido experiencia axiológica}

Vol. 2, núm. 2., (2018)

Ruth Cecibelt Cedeño Álvarez; José Bladimir Guarnizo Delgado; Iván Santiago Pacheco Proaño

En tanto la metodología empleada para esta experiencia estuvo signada por Ruesga (2011) que divide su propuesta en dos fases: la de proyección escénica, con seis pasos y la de ejecución escénica, con ocho pasos, en la que tomó partido la autora de esta investigación.

\section{Desarrollo.}

En el ámbito nacional se debe recordar que en la presentación del Plan Nacional del Buen Vivir (PNBV) de la República, el Secretario Nacional de Planificación y Desarrollo, explica las rupturas y los aportes que anunciaba el programa gubernamental para el período comprendido entre 2013 y 2017, poniendo énfasis en el eje referido a la revolución cultural:

Que genera nuevos conceptos y valores ciudadanos para constituir una sociedad crítica, emprendedora, creativa y solidaria. Se vuelven fundamentales las industrias culturales y la democratización de la palabra. También implica una revolución educativa para formar ciudadanos con otra mentalidad, con valores asentados, con ética y con autoestima. El Buen Vivir significa, sobre todo, tener en el país una población con una gran dosis de autoestima y de confianza colectiva. (Secretaria Nacional de Planificación y Desarrollo/Semplades, 2013, p. 18)

Esta experiencia axiológica del taller de teatro responde íntegramente a tal exigencia social que impone el país en la actualidad; la obra interpretada llevó a los espectadores un mensaje de no a la violencia.

Los participantes en general, -tanto personal activo vinculado a la puesta en escena como los consumidores de la obra-, lograron en debate espontáneo posterior al cierre del telón un taller donde críticamente expusieron la realidad de la nación y de cómo podían establecer una calidad 


\section{Adopción del recurso teatral en el tratamiento didáctico del proceso de enseñanza aprendizaje devenido experiencia axiológica}

Vol. 2, núm. 2., (2018)

Ruth Cecibelt Cedeño Álvarez; José Bladimir Guarnizo Delgado; Iván Santiago Pacheco Proaño de vida mejor, en el foro reunido prevaleció la confianza en la construcción de un Ecuador fuerte y seguro.

Además del comentario anterior, se debe señalar que esta experiencia estuvo estratégicamente diseñada para cumplir con varios de los objetivos del Plan Nacional del Buen Vivir, entre ellos estuvo: 3/ Mejorar la calidad de vida de la población; 4/ Fortalecer las capacidades y potencialidades de la ciudadanía; y 5/ Construir espacios de encuentro común y fortalecer la identidad nacional, las identidades diversas, la plurinacionalidad y la interculturalidad.

Al llevarles a los habitantes de la provincia de Bolívar una expresión artística como el teatro y el abordaje problematizador, se afirma contribuir a la mejora de calidad de vida; el pueblo demandó varias presentaciones más fuera del programa previsto, esto fue muestra de la pertinencia de la obra y del mensaje sensibilizador que la acompañó.

Es preciso añadir, se fortalecieron las capacidades y las potencialidades de los ciudadanos bolivarenses, debido a que se avanzó - con el ejercicio teatral y crítico de la expresión cultural -, hacia la sociedad del conocimiento anhelada por la mayoría en el territorio. Esta experiencia dedicó sus esfuerzos en garantizar todo el derecho constitucional de una educación con calidad y equidad, y puso en el centro de su actividad al ser humano y a la localidad.

También desde las tablas del escenario del teatro "Nilo" y de la Casa de la Cultura, de la provincia Bolívar, así como otras plazas culturales, se apreciaron espacios de encuentros comunes que potenciaron la identidad y los valores desde la promoción de una obra que respetó 


\section{Adopción del recurso teatral en el tratamiento didáctico del proceso de enseñanza aprendizaje devenido experiencia axiológica}

Vol. 2, núm. 2., (2018)

Ruth Cecibelt Cedeño Álvarez; José Bladimir Guarnizo Delgado; Iván Santiago Pacheco Proaño

la diversidad, la plurinacionalidad y la interculturalidad. Suponiendo esta línea de trabajo como

una sólida garantía del éxito alcanzado, no solo en lo artístico, sino en lo educativo axiológico.

La construcción de una identidad nacional en la diversidad tiene como requisito "la constante circulación de los elementos simbólicos que nos representan: las memorias colectivas e individuales y el patrimonio cultural tangible e intangible" (Semplades, 2013, p. 181) lo que está a tono con la exhibición de la obra. El coleccionista de senos.

El teatro tiene tres principios que se ajustaron a esta investigación y no pueden ser omitidos: primeramente, el teatro debe entenderse como un arte dramático, no puede alcanzar resultados de calidad estética si no intervienen todas sus partes en los continuos, prolongados y necesarios ensayos de diferentes tipos y muchos entendidos enfatizan en que el teatro es el espacio de las tres R: representación (espectáculo), recepción (espectador), repetición (ensayos) Brook, (2001).

Desarrolla la funcionalidad de la actividad, a través de interpretaciones de roles, tales como los de jerarquía (director), como los de subordinación (extras); los estudiantes en este nivel superior tienen la capacidad para comprender íntegramente las funciones comunicativas de la dramaturgia, los signos y símbolos fueron apreciados por ellos y trasmitidos desde la escena.

Como otro de los principios del teatro se manifestó como materia de enseñanza, el valor cognoscitivo del arte apreciado no sólo en el proceso de hacerlo y de verlo, sino además de trasmitirlo y de apreciarlo, ya que los estudiantes crearon, estudiaron y elaboraron propuestas en cada uno de los momentos de la puesta en escena, esto fue muestra del interés y la motivación, signada por el compromiso de presentar un buen espectáculo y de que el público presente en las 


\section{Adopción del recurso teatral en el tratamiento didáctico del proceso de enseñanza aprendizaje devenido experiencia axiológica}

Vol. 2, núm. 2., (2018)

Ruth Cecibelt Cedeño Álvarez; José Bladimir Guarnizo Delgado; Iván Santiago Pacheco Proaño salas de exposición se llevase el mensaje contra la violencia. Sobre esta etapa educativa se confía en que "hay que optar por enfoques más flexibles y didácticamente más ricos, centrados en el proceso de investigación y aprendizaje, en el proceso de compartir y aportar ideas y en el proceso de creación" (Motos, 2009, p.7)

Casi vinculado a este principio intermedio está el tercero, el cual se manifiesta como un componente de alfabetización artística. Para que la generalidad de los integrantes del taller alcanzase esta condición, tuvieron que ver o leer teatro, debatir y comunicarse con los cánones del lenguaje teatral, asumir las capacidades o competencias necesarias que generaron una posición activa y reflexiva, fueron capaces de disfrutar de la obra y valorarla a plenitud.

El artículo del referido Tomás Moto (2009) defiende la tesis de que el teatro se ha convertido para la educación en un medio conveniente para alcanzar la integración de los contenidos y las experiencias curriculares, promovido esencialmente porque se trata de un lenguaje total, este trabajo se convirtió en referencia y guía para poner en marcha esta vivencia. La diferencia más marcada entre el trabajo del español y el resultado descrito en estas páginas está en la existencia de dos usuarios con diferencias generacionales bien definidas, además de las distancias culturales y sociales entre un país europeo y una nación multicultural como el Ecuador, que presenta un proyecto de inclusión favorecedor del arte y de la educación.

Estos autores evalúan cuatro retos, los que fueron adecuados a la experiencia axiológica de los estudiantes de Comunicación Social, ellos están determinados como: consolidar la inserción del teatro en el currículum; para cumplir con esto se trazó una estrategia didáctica, se 


\section{Adopción del recurso teatral en el tratamiento didáctico del proceso de enseñanza aprendizaje devenido experiencia axiológica}

Vol. 2, núm. 2., (2018)

Ruth Cecibelt Cedeño Álvarez; José Bladimir Guarnizo Delgado; Iván Santiago Pacheco Proaño incorporó como contenido a la asignatura de Lenguaje y Comunicación y se desarrolló como actividad teatral; Definir las competencias en educación teatral, las cuales fueron agrupadas y tratadas en esta experiencia.

Otro reto alcanzado, la formación del profesorado como artista pedagogo y como mediador, este reto fue asumido por un grupo de docentes de la U/E. Bolívar asesorados por profesionales del teatro vinculados a la Casa de la Cultura de la provincia y por último el eliminar las barreras de acceso de los jóvenes al teatro, en este aspecto se utilizaron los fundamentos teóricos de los autores norteamericanos McCarthy \& Jinnett (2001).

Sobre la base de estos retos se profundizó en la descripción de la experiencia docenteeducativa que abarcó los espacios universitarios y culturales de la provincia bolivarense. Se tuvo en cuenta en la lógica descriptiva la importancia del currículum, las competencias de dramatización y la eliminación de barreras para la asistencia al espectáculo teatral.

Se indaga aplicando las herramientas heurísticas sobre la importancia y la pertinencia de incluir el teatro como parte del currículum, particularmente dentro de la disciplina Lenguaje y Comunicación; no se entendía cómo dentro de la identificación de los contenidos y de las prácticas educativas esta manifestación artística emergería con algún valor.

Al no existir ningún precedente en la U/E. Bolívar o en otras casas de altos estudios del territorio que incluyeran al teatro dentro de la formación curricular de los estudiantes, se trazó como camino, hacer un recorrido por la teoría, encontrando textos de diferentes puntos geográficos que apoyaban la idea. Estos autores están relacionados en la introducción de este artículo pues aprecian las claves del arte dramático en el proceso de enseñanza y aprendizaje en 


\section{Adopción del recurso teatral en el tratamiento didáctico del proceso de enseñanza aprendizaje devenido experiencia axiológica}

Vol. 2, núm. 2., (2018)

Ruth Cecibelt Cedeño Álvarez; José Bladimir Guarnizo Delgado; Iván Santiago Pacheco Proaño los temas enunciados como es el comprometimiento, por parte de los estudiantes, con la vivencia teatral-cognoscitiva, así como el mejoramiento de la comunicación y el lenguaje en los educandos.

Los estudiantes desarrollan rasgos de la personalidad como: la creatividad, la independencia, la auto-crítica, entre otro, logrando habilidades particulares y se fomenta la interdisciplinariedad con otras manifestaciones artísticas. Resumiendo, estos enfoques el profesor valenciano Tomás Motos (2009) escribió:

La dramatización y las estrategias didácticas teatrales por su carácter transversal e interdisciplinario se revelan como un instrumento didáctico eficaz para desarrollar aspectos de las competencias básicas y especialmente: competencia en comunicación lingüística; competencia cultural y artística; competencia social y ciudadana; competencia para aprender a aprender; y competencia en autonomía e iniciativa personal. (p. 9)

En los próximos párrafos se ejemplifican las competencias de dramatización (tanto de ejecución como de creación) que se utilizó en la obra de teatro, estas fueron adaptadas del artículo de Motos (2009, pp. 15-18).

En relación a la competencia de expresión corporal: el villano, Don Mariano, encontró la forma de acentuar su lado oscuro con un estilo particular de caminar en escena, con estos gestos enfatizó su interpretación, logrando el efecto deseado en el público de desprecio hacia el personaje que caracterizaba el actor-estudiante. 


\section{Adopción del recurso teatral en el tratamiento didáctico del proceso de enseñanza aprendizaje devenido experiencia axiológica}

Vol. 2, núm. 2., (2018)

Ruth Cecibelt Cedeño Álvarez; José Bladimir Guarnizo Delgado; Iván Santiago Pacheco Proaño

En cuanto a la competencia de expresión oral se pudo apreciar en la manera que utilizó el galán de la obra, Don José, en sonorizar los poemas que declaró a su enamorada, de tal forma que proyectaba adecuadamente la voz, como uno de los instrumentos principales de un artista, en el espacio teatral, empleando una articulación y dicción acertadas, tal y como lo demandaba su arquetipo. Igualmente, el narrador desde una voz en off encontró la manera de realzar los ambientes y situaciones con su descripción.

En tanto la competencia de improvisación estuvo presente en varios momentos del texto, quizás el más aclamado fue donde la joven María tuvo que recurrir a su memoria emocional y representar gestos y expresiones corporales (voz, movimientos espaciales) de una víctima.

El uso de los elementos y estructuras del lenguaje dramático como competencia se manifestó en la gestión del director de la obra cuando planificó la representación de las escenas con coherencia, además de velar por las cualidades técnicas y estéticas de la representación consiguiendo un resultado aceptable.

La competencia de composición dramática: composición individual y colectiva se evidenció con la participación de manera constructiva en el trabajo del grupo tallerista, al proponer y tomar las iniciativas.

Por último, la competencia de contextualización, análisis y valoración, se demostró cuando el nivel teórico del taller permitió la adquisición de criterios para comprender cómo han cambiado las manifestaciones teatrales a lo largo del tiempo y como se relacionan con otros ámbitos (ciencia, religión, sociedad, arte). 


\section{Adopción del recurso teatral en el tratamiento didáctico del proceso de enseñanza aprendizaje devenido experiencia axiológica}

Vol. 2, núm. 2., (2018)

Ruth Cecibelt Cedeño Álvarez; José Bladimir Guarnizo Delgado; Iván Santiago Pacheco Proaño

En otras competencias (Motos, 2009, p. 21) expresa "en este caso las de transferir los fundamentos teóricos a la práctica educativa, se realizó el mismo intento de ejemplificación, ahora con el protagonismo del docente encargado de la puesta en marcha de la obra teatral", advirtiendo que el resultado fue cooperación entre los docentes de Lenguaje y Comunicación, Escenografía y otras disciplinas afines estudiadas en la U/E. Bolívar.

La competencia de aprobación estuvo de manifiesto en el establecimiento de los lazos entre el arte y la pedagogía, así como en el constante desvelo por la auto preparación académica. En tanto, la competencia de adaptación tuvo en el uso de la improvisación teatral y pedagógica y en el análisis reflexivo de la práctica su afirmación.

De la misma manera que la competencia de creación se materializó en la capacidad de utilizar la creación individual de cada uno de los implicados en la experiencia como en la creación colectiva del Taller.

En último término, la competencia de definición interesó el rol de cada uno de los estudiantes y docentes involucrados en el proceso y resultado docente-teatral, mediante la definición de los diferentes roles: autor, actor, director, técnico, crítico, espectador, etc.

En esta parte del artículo se procede a explicar la experiencia pedagógica vivida en la ciudad de Chimbo, provincia de Bolívar, a través del modelo sobre decisiones individuales para participar en actividades culturales que patentaron McCarthy \& Jinnett (2001) el cual comprende cuatro fases. 


\section{Adopción del recurso teatral en el tratamiento didáctico del proceso de enseñanza aprendizaje devenido experiencia axiológica}

Vol. 2, núm. 2., (2018)

Ruth Cecibelt Cedeño Álvarez; José Bladimir Guarnizo Delgado; Iván Santiago Pacheco Proaño

La fase inicial denominada de antecedentes comprendió los factores socio-demográficos, tales como: nivel educacional, ingresos personales y familiares, género y edad, entre otros, el factor personológico; las experiencias anteriores en relación al arte en general y al teatro en particular y en cuarto puesto los socio-culturales que tuvieron que ver con la identidad o pertenencia del individuo a determinado grupo social o étnico-cultural.

Como regularidad se estableció una disposición casi nula del grupo a iniciar la experiencia, esto estaba manifestado porque planteaban que el teatro no era parte de sus vidas recreativas, de estudio o de ocio. Los que tenían una inclinación positiva a este arte confesaban en las entrevistas que eran presionados por el resto de sus amigos que no tenían el hábito de la actividad teatral.

Por otra parte, en una búsqueda en documentos normativos, currículum y otras fuentes se apreció que la sede universitaria de Bolívar estaba despoblada de iniciativas como la que llevó a cabo el IV ciclo de la carrera de Comunicación Social. En relación a las experiencias familiares entorno al teatro también eran escasas.

En la fase siguiente llamada perceptual se comprobó que los estudiantes tenían una predisposición a la actividad teatral, dada en primer lugar por las creencias personales sobre la participación en las artes y en segundo término por la percepción de las normas sociales hacia estas actividades, ello estaba íntimamente relacionado con las actitudes individuales participativas.

Como regularidad se escucharon opiniones desfavorables, tales como: "el teatro no tiene que ver conmigo; es para personas de edades avanzadas; es aburrido; hay otras manifestaciones 


\section{Adopción del recurso teatral en el tratamiento didáctico del proceso de enseñanza aprendizaje devenido experiencia axiológica}

Vol. 2, núm. 2., (2018)

Ruth Cecibelt Cedeño Álvarez; José Bladimir Guarnizo Delgado; Iván Santiago Pacheco Proaño superiores en rango de preferencia (música, cine, videos juegos, etc.); el buen teatro solo se produce en la capital del país.”

Sin embargo, no todo lo recogido fue penoso, también se escucharon criterios o razones para asistir a una actividad teatral: interés por el espectáculo (la actualidad y la identificación con el tema de la obra); la oportunidad de interacción social; el interés cognoscitivo visto como fuente de enriquecimiento personal. Algunos más osados y comprometidos plantearon la necesidad de expresarse artísticamente y manifestaron la responsabilidad para con sus compañeros y con la comunidad.

La tercera fase de carácter práctico consistió en la estimación individual sobre las oportunidades reales de participar y la decisión de asistir al espectáculo, los obstáculos que impedían la concreción de la actividad giraban alrededor de la disponibilidad de presupuesto y/o de tiempo, la distancia (traslación), los horarios y los intereses juveniles plasmados en escena.

Los docentes y un grupo de estudiantes voluntarios con disposición a lograr una experiencia pedagógica-teatral de elevada calidad se prestaron a revertir la situación. Para ello se trazó una estrategia que promocionó los beneficios del arte y del teatro, se desarrollaron actividades docentes vinculadas a la obra teatral de importantes dramaturgos nacionales y del resto de Iberoamérica.

Este movimiento universitario pro-teatral tuvo como objetivo la inclusión de la mayoría de los estudiantes a la experiencia de la puesta en escena de "El coleccionista de senos". Con el transcurso del tiempo y la celebración del taller como espacio aglutinador de los interesados, llegaron los resultados esperados: la participación masiva espontánea de los estudiantes. 


\section{Adopción del recurso teatral en el tratamiento didáctico del proceso de enseñanza aprendizaje devenido experiencia axiológica}

Vol. 2, núm. 2., (2018)

Ruth Cecibelt Cedeño Álvarez; José Bladimir Guarnizo Delgado; Iván Santiago Pacheco Proaño

Al llegar a la última fase comprendida como la experiencia en sí,-per se-, los involucrados derribaron las barreras y tabúes en relación con el teatro, aquí se apreció no solo el momento de la participación activa como gestor de la obra o activa-pasiva como espectador y crítico de la misma; sino el momento posterior dirigido a valorar la experiencia y extraer las mejores enseñanzas, este espacio para las reacciones fue favorecido por el ambiente de camaradería que acompañó al grupo de estudiantes durante las semanas que duró el proceso.

En el trabajo de Monserrat González Parra (2012) se establece un modelo formativo a través del teatro en donde figuran cinco momentos de la práctica teatral. Esta investigación fue tomada en cuenta por el taller de la U/E. Bolívar. Para una mejor comprensión se referencia este aporte: 1/ Conciencia de la temática o el texto que interesa tratar; 2/ Sentir, experimentar la información como algo que interesa y nos afecta; 3/ Pensar el tema con los agentes o actores en términos de acción; 4/ Aplicar, ejecutar, llevar a la práctica la propuesta escénica; 5/ Integrar y trascender. (p. 52)

Son un quinteto de operaciones sensoriales que sirven de porteras a las emociones y al conocimiento, cada una de ellas con una prolongación temporal variable en dependencia de la práctica.

En el primer paso de tomar conciencia se logró interesar a los participantes en el problema del aumento de la violencia y, por ende, en la búsqueda de una sociedad más justa y solidaria. En este momento de la experiencia los estudiantes al tomar conciencia de la realidad como premisa para alcanzar el conocimiento declararon lo que querían hacer y para qué, 


\section{Adopción del recurso teatral en el tratamiento didáctico del proceso de enseñanza aprendizaje devenido experiencia axiológica}

Vol. 2, núm. 2., (2018)

Ruth Cecibelt Cedeño Álvarez; José Bladimir Guarnizo Delgado; Iván Santiago Pacheco Proaño desarrollándose un importante debate sobre el mensaje de la obra. Ritual, que posteriormente se realizó en cada jornada previa a comenzar los ensayos de la obra.

En cuanto a sentir, entiéndase lo referente a las emociones y valores, estas desempeñan un papel de vanguardia en el proceso de aprendizaje y de transformación de la personalidad, por lo que en la práctica se sensibilizó a los integrantes del taller en la lucha contra la violencia, una vez asumida esta conducta pudieron llevar al público, a través de la representación teatral escogida, el mensaje deseado.

Pensar y planificar la acción estuvo a cargo de los líderes de la puesta en escena: director, autor, productor, escenógrafo y actores protagonistas, ellos en trabajo de mesa elaboraron cada idea y tomaron las principales decisiones, el resto del personal fue involucrado en cada decisión y tomado en cuenta si sus proposiciones eran avaladas por la mayoría.

En el momento de la aplicación los estudiantes integraron emociones y conocimiento, paso imprescindible para que los valores quedaran interiorizados en cada uno de ellos.

El último momento de este modelo formativo correspondió a la integración y la trascendencia de la acción educativa-teatral. El teatro tiene la capacidad de trasladar al público a la realidad que vive, mostrándola en un escenario por actores que invitan a la reflexión individual teniendo como base situaciones previas de la vida cotidiana, en este sentido los que concurrieron a cada presentación de la obra se comprometieron con la causa de erradicar la violencia; de crear una ciudad pacífica. 


\section{Adopción del recurso teatral en el tratamiento didáctico del proceso de enseñanza aprendizaje devenido experiencia axiológica}

Vol. 2, núm. 2., (2018)

Ruth Cecibelt Cedeño Álvarez; José Bladimir Guarnizo Delgado; Iván Santiago Pacheco Proaño

El resultado de la investigación de la autora catalana González (2012) es dirigido a los

profesionales de la educación, queda por debajo de las expectativas en puntos como el marco teórico, en la caracterización del teatro como elemento emisor de enseñanzas y de otras funciones.

En cuanto al modelo de formación asumido en este artículo fue considerado como pertinente para la aplicabilidad de la experiencia del taller de la U/E. Bolívar, la novedad fue que se interesó no solo a los docentes sino a un grupo de estudiantes y a sus familiares, se centró la actividad hacia una acción axiológica determinada.

En cuanto a la metodología aplicada a esta experiencia axiológica estuvo determinada por la propuesta de Ruesga Navarro (2011). Ella comprendió las dos fases proyectadas para la realización de un espectáculo teatral. Primera: la fase de proyección escénica, con seis pasos y segunda: la fase de ejecución escénica, con ocho pasos.

Todos fueron acatados y adaptados a las exigencias de un taller pequeño en número de participantes y en presupuesto concebido, en la primera fase los pasos fueron los siguientes: (1ro.) idea del espectáculo; (2do.) proyecto dramatúrgico; (3ro.) presentación del proyecto dramatúrgico a los diseñadores escénicos y al resto del área artística; (4to.) anteproyecto de los diseñadores; (5to.) reunión de coordinación de los anteproyectos. Productor artístico. Director de puesta en escena y (6to.) ensayos preliminares. Sesiones de debate con el dramaturgo, director de puesta en escena, productor y todo el equipo de diseño y el resto del área artística. Fijación de criterios para la realización. (pp. 98-100) 


\section{Adopción del recurso teatral en el tratamiento didáctico del proceso de enseñanza aprendizaje devenido experiencia axiológica}

Vol. 2, núm. 2., (2018)

Ruth Cecibelt Cedeño Álvarez; José Bladimir Guarnizo Delgado; Iván Santiago Pacheco Proaño

En la segunda fase y definitoria del resultado para el cual se trabajó durante mucho tiempo comprendió los pasos a continuación enlistados: (1ro.) los proyectos de ejecución. Reunión preliminar con el director técnico y el productor; (2do.) finalización de los proyectos de ejecución. Reunión de control con el director técnico y el productor; (3ro.) realización / construcción de los elementos; (4to.) implementación de los elementos en el escenario; (5to.) ensayos en el escenario; (6to.) ensayos técnicos; (7mo.) ensayos generales y (8vo.) preestrenos con público. (pp. 100-106)

Esta metodología para lograr un espectáculo teatral fue de singular importancia para la realización y puesta en escena de la obra: El coleccionista de senos, proyectada por el Taller de la U/E. Bolívar, en el mes de julio de 2014.

\section{Conclusiones.}

La inclusión del teatro en el proceso de enseñanza y aprendizaje de la asignatura: Lenguaje y Comunicación a través de la puesta en escena de la obra: El coleccionista de senos, como parte del desarrollo de un taller teatral dentro del currículum de los estudiantes del IV ciclo de la carrera de Comunicación Social propició una experiencia axiológica en la U/E. Bolívar y en el resto de la ciudad de Chimbo, provincia de Bolívar, Ecuador.

La implementación de un modelo formativo dedicado a los estudiantes universitarios permitió sensibilizarlos en relación a la lucha contra la violencia. Igualmente, la exportación de una metodología estructurada en dos fases (proyección y ejecución escénica) para aplicar al taller de creación y estudio teatral fue bien recibida por la sociedad bolivarense en tanto fomentó una cultura contra la violencia, lo que determinó la pertinencia de esta experiencia axiológica. 


\section{Adopción del recurso teatral en el tratamiento didáctico del proceso de enseñanza aprendizaje devenido experiencia axiológica}

Vol. 2, núm. 2., (2018)

Ruth Cecibelt Cedeño Álvarez; José Bladimir Guarnizo Delgado; Iván Santiago Pacheco Proaño

\section{REFERENCIAS BIBLIOGRÁFICAS:}

Andrew P, J. (2000). The Perfect Tools for Gifted Students. Gifted Child Today, 23(2), 30-33.

Aparicio, G. (2009). La Educación en Valores a través del teatro. Hekademos: Revista Educativa Digital, 2(1), 85-98.

Ausubel, D. (1975). Psicología Educativa. México: Trillas.

Baldwin, P., Fleming, K., \& Neelands, J. (2003). eaching Literacy through Drama: Creative Approaches. London: Routledge Falmer.

Brook, P. (2001). El espacio vacío. Arte y técnica del teatro. Barcelona: Península.

Froese, V. (1996). Whole-Language: Practice and Theory. Needham: ERIC.

González Parera, M. (2012). El teatro como estrategia didáctica. Madrid: Instituto Cervantes.

Laferriére, G., \& Motos, T. (2003). Palabras para la acción. Términos de teatro en la educación y la intervención sociocultural. Ciudad Real: Naque.

McCarthy, K., \& Jinnett, K. (2001). A New Framework for Building Participation in the Arts. Santa Monica: RAND Corporation.

Meléndez Viana, S. (2013). Fundamentos actuales para una metodología de la puesta en escena. México D.F.: Secretaria de Cultua de México.

Motos, T. (2009). El teatro en la educación secundaria: fundamentos y retos. Creatividad y Sociedad, 1-35.

Navarro Solano, M. (2006). El valor pedagógico de la dramatización su importancia en la formación inicial del profesorado. Creatividad y sociedad: revista de la Asociación para la Creatividad, 19(206), 11-18.

O'Neill, C. (1995). Drama Worlds: A Framework for Process Drama (The Dimensions of Drama). Portsmouth: Heinemann.

Ruesga Navarro, J. (2011). Metodología de la Plástica Escénica La producción artística. Anagnórisis, 4(1), 88-109.

SENPLADES. (2013). Plan Nacional del Buen Vivir 2013-2017. Quito: autor.

Vizcaino, J. (2015). El coleccionista de senos. Chimbo, Bolívar, Ecuador. Quito: Manuscrito no publicado. 


\section{Adopción del recurso teatral en el tratamiento didáctico del proceso de}

enseñanza aprendizaje devenido experiencia axiológica Vol. 2, núm. 2., (2018)

Ruth Cecibelt Cedeño Álvarez; José Bladimir Guarnizo Delgado; Iván Santiago Pacheco Proaño Vygotsky, L. (1978). Psicología del Arte. Barcelona: Paidós Iberia.

Wagner, B. (1998). Educational Drama and Language Arts. Portsmouth: Heinemann. 